\title{
Desarrollo de la modelación tecnológica a partir del uso de videojuegos ${ }^{1}$
}

\author{
Jorge Hernán Alvarez Pérez ${ }^{2}$
}

\begin{abstract}
Resumen
En este documento se presenta una investigación desarrollada en el año 2011-2012 acerca de la aplicación de los videojuegos en el desarrollo de habilidades para la modelación en tecnología, en los temas de electricidad y electrónica, a partir de la construcción de un espacio lúdico que permitiera el trabajo con los videojuegos Liquid Measured donde el objetivo es tomar decisiones para no desperdiciar ni una gota de aguay Cargo Bridge para la construcción de puentes a partir de la asignación de un presupuesto limitado y el análisis de estructuras, buscando llevar los conocimientos de las ciencias básicas y aplicarlos en contextos reales, para unir de esta manera la teoría con la práctica. Este estudio fue aplicado a 44 estudiantes con edades comprendidas entre los 14-17 años, divididas en 22 estudiantes para el grupo de control y 22 estudiantes para el grupo experimental. Los resultados obtenidos muestran un mejor desempeño en la prueba de modelación del grupo experimental con respecto al grupo de control, además se logra evidenciar el interés de las estudiantes por alcanzar los diferentes niveles que debían resolver en cada uno de los videojuegos; el trabajo colaborativo se convirtió en una oportunidad de aprendizaje en el aula de clase que llevaba a las estudiantes a proponer alternativas, plantear ideas frente a cada una de las situaciones que se presentaban en los diferentes niveles que debían solucionar, además se logró evidenciar durante el trabajo desarrollado en la investigación un excelente ambiente de aula que facilito el desarrollo de las actividades.
\end{abstract}

Palabras claves: modelación, creatividad, motivación, lúdica, videojuego

\section{Development of modeling technology from the use of video games}

\begin{abstract}
This paper presents research developed in 2012 on the implementation of video games to develop skills in modeling technology in the areas of electricity and electronics, from the construction of a recreational space that would allow the work with the video games Measured Liquid, where the goal is to

1 Recibido: 28 de marzo del 2012. Aceptado: 03 de mayo del 2012.

2 Jorge Hernán Álvarez Pérez. Ingeniero Informático politécnico Colombiano Jaime Isaza Cadavid. Estudiante de Maestría en Tecnología Educativa y Medios Innovadores para la Educación de la Universidad Tecnológico de Monterrey y Unab, México. Docente del Colegio Bethlemitas, Fundación Universitaria Luis Amigo y Fundación Universitaria Autónoma de las Américas, Correo Electrónico: jalvarezp243@gmail.
\end{abstract} com 
make decisions for not to wasting even a drop of water, and Cargo Bridge, for bridge construction from the allocation of a limited budget and analysis of structures, seeking to apply the basic science knowledge in real contexts to unite this way theory and practice. This study was applied to 44 students aged between 14 to 17 years old, divided into 22 students for the control group and 22 students for the experimental group. The results obtained show a better performance in the test modeling of the experimental group compared to the control group. In addition to this, it was also possible to demonstrate the interest of students to achieve the different levels that were solved in each game. Collaborative work became a learning opportunity in the classroom that led the students to propose alternatives and suggest ideas for the situations that were presented at different levels to be solved. Likewise, it was possible to see an excellent academic atmosphere during the research that made the development of the classroom activities lighter.

\section{Antecedentes}

Se puede llegar a pensar que el juego se emplea para educar a niños pequeños, ya que es en los primeros grados de estudio (preescolar) es donde los estudiantes tienen un gran deseo y disfrutan de este, como una estrategia lúdica de aprendizaje, a medida que los estudiantes crecen no ven el juego como una oportunidad para aprender, ya que sienten que son muy mayores para realizar determinado tipo de actividades (les avergüenza).

"Esta situación se hace comprensible, debido a que en algunas sociedades, se considera que jugar es una actividad únicamente para niños y que cuando los adolescentes y adultos la practican, no logran mucho" (Palacino, Fredy 2007).

El juego es un medio para el desarrollo del aprendizaje y la comunicación que no tiene en cuenta las edades, pero que es una fuente de placer, diversión, que lleva al que juega a sentirse libre para tomar decisiones de cómo va a jugar, que herramientas va a emplear, además de respetar una normatividad que debe seguir, estas características facilitan la creación de hábitos, para convivir y compartir en una sociedad regida por reglas y normas.

Las experiencias en contextos educativos han llevado a demostrar que los videojuegos permiten desarrollar competencias en los estudiantes, tal es el caso de:
Lacaso, Méndez y Martínez (2008), quienes estimulan el desarrollo de habilidades comunicativas con el juego de Harry Potter, Bernat (2008) Emplea el juego Age of Empire en un curso de 6 y encontró que las competencias que se adquieren son las siguientes:

1). Competencias para la gestión de recursos, entre las que especifica: a. competencias para la gestión de la información, y b. competencias para la gestión de los recursos digitales: buscadores, correo electrónico, editores gráficos, enciclopedias digitales, etc., entre otras. 2). Competencias para la comunicación. En este grupo señala: a. competencias para la comunicación por medios electrónicos, y con las características propias: hipertextualidad, multimodalidad, inmediata e interactiva. Y b. competencias para la comunicación oral reflexiva, argumentada y situada. 3 ). Competencias para la crítica reflexiva relacionadas con una actitud responsable en las sesiones de juego en cuando a las normas de juego que se establecen y los pactos que se negocien.

(Lave, 1988), Todos los días la experiencia es la mejor manera por la cual las culturas inciden en los individuos y viceversa, la funcionalidad de la practica social implica diferentes preguntas a respuestas acerca de la apropiación cognitiva de la actividad como objeto de análisis. 
(Carraher y Schliemann, 1991), EI aprendizaje de las matemáticas en el salón de clases es un momento de interacción entre las matemáticas organizadas por una comunidad científica, o sea, las matemáticas formales, y las matemáticas como actividad humana. En primer lugar no debemos olvidar que el profesor es una persona que organiza su propia actividad matemática. Es importante destacar que las matemáticas que se practican en el salón de clases son una actividad porque lo que interesa en esta situación es el aprendizaje del alumno, lo que debe llevar al sujeto a una construcción de su conocimiento. Que lo debe llevar a establecer relaciones entre los objetos de nuestro conocimiento, para contarlos medirlos, sumarlos, dividirlos, etc, y verificar los resultados de diferentes formas de organización que escogemos para nuestras actividades.

La importancia de la práctica y la experimentación de los conceptos por parte de los estudiantes, les permitirá establecer conexiones entre los objetos y otros con los que han interactuado.

Tal es el caso de (Lave, 1988), Al realizar el estudio sobre los circuitos eléctricos en serie y en paralelo, los estudiantes realizaban preguntas acerca de las corrientes en cada una de las configuraciones y establecían similitudes con los corrientes de agua, esto les permitía tener una mejor asimilación del fenómeno que estaban estudiando.

Todas estas necesidades de aprendizaje además de la aplicación de mejores metodologías de enseñanza, llevan a analizar las dificultades que se encuentra en las estudiantes del grado Decimo a la hora de modelar, o diseñar una solución a un problema que implique la aplicación de los conocimientos teóricos en las áreas de ciencias básicas y tecnología en una situación real, la cual las debe llevar a manipular los conceptos adquiridos en clase, además de diseñar los respectivas modelos computacionales que deben dar solución al problema planteado, y además que disfruten y gocen del desarrollo de competencias y nuevos conocimientos.

Basado en la problemática anterior, se desea aprovechar las estrategias lúdico virtuales como una propuesta que permita atraer la atención, el interés y la pasión por desarrollar las competencias de modelar, diseñar estrategias para la solución de problemas de ciencias básicas y tecnología, aprovechando el gusto que tienen los estudiantes por los juegos, y en especial por los video juegos mirados como una herramienta que permite desarrollar aspectos cognitivos tales como: "Memorización de hechos, Observación hacia los detalles, Percepción y reconocimiento espacial, Descubrimiento inductivo, Capacidades lógicas y de razonamiento, Comprensión lectora y vocabulario, Conocimientos geográficos, históricos, matemáticos, Resolución de problemas y planificación de estrategias."(Grupo F9; Gree, 2004; Alfageme, 2003).

Teniendo presente los aspectos cognitivos que desarrollan los video juegos, tales como: lógica, razonamiento espacial, memoria, resolución de problemas, toma de decisiones, se tienen como una de las estrategias lúdicas virtuales, que facilitaran el desarrollo de competencias de las estudiantes en el área de Tecnología y ciencias básicas, a la hora de modelar y diseñar problemas reales y aplicados en un determinado contexto.

\section{Problema}

¿Cómo los videojuegos facilitan el desarrollo de competencias en modelamiento científico y tecnológico en las estudiantes de bajo nivel académico?

\section{Objetivo general}

Determinar la efectividad del uso de video juegos en el desarrollo de competencias que permitan mejorar la habilidad de las estudiantes para solucionar problemas tecnológicos. 


\section{Objetivos específicos}

Identificar la percepción de las estudiantes sobre el aprendizaje basado en video juegos aplicados en los espacios de aprendizaje.

Diseñar estrategias que permitan la construcción de modelos computacionales empleando video juegos.

Diseñar ambientes de aprendizaje que motiven la construcción de prototipos tecnológicos donde se permita unir la teoría de la ciencia básica con la práctica, aplicada a situaciones de la vida cotidiana.

Utilizar estrategias lúdicas apoyadas por video juegos, para facilitar y motivar el desarrollo de actividades que implican la abstracción de situaciones del mundo real.

\section{Descripción teórica}

El videojuego es considerado una herramienta potencial en la educación, objeto de estudio en la psicología del comportamiento, una herramienta para la interacción social, un juguete y medio de distracción.

Las características antes mencionadas convierten al videojuego en una estrategia para el aprendizaje, que apoyado de la lúdica busca que los usuarios que lo utilicen logren divertirse, al mismo tiempo que desarrollan aspectos cognitivos tales como: "Memorización de hechos, observación hacia los detalles, percepción y reconocimiento espacial, descubrimiento inductivo, capacidades lógicas y de razonamiento, competencia lectora y vocabulario, conocimientos geográficos, históricos y matemáticos, Resolución de problemas y planificación de estrategias".(Grupo F9; Gree, 2004; Alfageme, 2003).

Entre las destrezas y habilidades, se pueden encontrar: "Autocontrol y autoevaluación, Motivación, Instinto de superación, habilidades motrices de reflejo y respuestas rápidas, percepción visual, percepción espacial, curiosidad e inquietud por probar e investigar" (Grupo F9; Gree, 2004; Alfageme, 2003).

Entre los aspectos socializadores, que se pueden desarrollar se encuentran: "Aumenta la autoestima: proporcionan un sentido de dominio, control y cumplimiento. Debido en gran parte a que existen recompensas personalizadas" (Grupo F9; Gree, 2004; Alfageme, 2003), otro tipo de habilidades que desarrollan son: las interpersonales y habilidades cooperativas, las primeras se destacan en la interacción en red, cuando el individuo debe aplicar competencias conversacionales, y asertivas definida como: "la capacidad de un individuo para transmitir sus posturas, opiniones, creencias o sentimientos de manera eficaz y sin sentirse incómodo" (Kelly, 1992). Las segundas se presentan como habilidades de comunicación y habilidades de controversia. (Lobato, 1998), plantea dos clases de habilidades cooperativas, habilidades ligadas a la tarea del grupo y habilidades ligadas a la relación de los miembros del grupo para poder desarrollar la tarea propuesta. Y son las habilidades de relación las que más pueden ayudar a potenciar los videojuegos, llevando al individuo a interactuar con mayor facilidad con las personas que convive o con las que debe trabajar.

Como instrumento didáctico "Los videojuegos proporcionan nuevas formas de aprendizaje e información, además de entretener y distraer. Potencian, también, la concentración y la atención, además de ayudar a construir las habilidades físicas y psicomotoras que el niño y del adolescente necesitan en esa etapa de su vida." (Marín y García, 2005, 117).

Es por tal motivo que el aporte de los videojuegos en el ámbito educativo, se presenta como una forma de aprender a aprender, tal como lo expone Greenfield, 1989, los estudiantes con dificultades de aprendizaje a la hora de concentrarse en el desarrollo de alguna actividad, prestaban atención al videojuego y lograban alcanzar las metas propuestas, Etxeberría, 2000 , demuestra que los videojuegos son 
de gran ayuda en el tratamiento de problemas educativos y terapéuticos, además de problemas psicológicos y físicos, esto hace que los videojuegos sean empleados en el campo de la reeducación, en el cual se han realizado numerosas investigaciones que en la mayoría de los casos han presentado resultados satisfactorios, tal como lo plantea Etxeberría, 2001, los videojuegos aplicados a las siguientes áreas: habilidades de relación, comunicación entre niños, trastornos del lenguaje, reducción de conductas antisociales, conductas impulsivas, aumento del autocontrol en jóvenes delincuentes, reducción de conductas autodestructivas, desarrollo de la cooperación, reducción de la ansiedad, toma de decisiones respecto a las drogas, regulación de la tensión arterial y presión sanguínea.

\section{Videojuegos y las aplicaciones educativas}

A continuación se presentan una serie de videojuegos en los cuales se muestran las inteligencias o habilidades que se pueden trabajar.

(Málaga, 2009). Los juegos tipo Arcade, en los cuales no se planifica ninguna acción, sino que se interactúa con el entorno lo más rápido posible, entre estos podemos encontrar Spacelnvaders, Tekken, Mortal Kombat, Doom, Quake, Halo, Call of Duty, son utilizados como actividades de entrenamiento para las fuerzas militares

Juegos de estrategia, en estos se hace necesario plantear estrategias para poder avanzar en el juego, con lo que se desarrolla el pensamiento lógico y la resolución de problemas, entre ellos tenemos: Civilization IV, Age of Empire, en estos juegos se trata de avanzar por las diferentes civilizaciones.

El juego Starcraft, su potencial educativo ha sido analizado por la Universidad de berkeley en california, en la cual se descubrió el potencial de ayudar a los estudiantes a entender estrategias bé- licas, a partir del estudio de ecuaciones diferenciales.

EI Juego UFO (EnemyUnknow), se aplican estrategias socio económicas, para aprender a invertir en capital humano.

Videojuego Rise of Nations, ha sido objeto de estudios (Kramer, 2008) Citado por (Malaga, 2009). En lo que se ha concluido que es de gran utilidad a las personas mayores a la hora de mejorar la velocidad y la habilidad para cambiar de tareas, además se observa que mejora la capacidad de razonamiento y la memoria aplicada a la resolución de alguna actividad.

Juegos de aventura, en este tipo de juegos se hace necesario estar tomando decisiones de forma constante, entre ellos podemos encontrar: King Quest, TheSaret of Monkey, Indiana Jones.

Juegos deportivos, este tipo de juegos permiten desarrollar habilidades de procesamiento de información y el desarrollo de la senso - motricidad, entre estos juegos se puede encontrar: FIFA, Pc Footbal, NBA, Formula I GrandPrix.

Juego de rol, tiene gran similitud a los juegos de aventura, pero en vez de dedicarse a la resolución de enigmas, se preocupa por la evolución de los personajes, entre ellos podemos encontrar Final Fantasy, NeverWinterNights, DeadSpace, Oblivion, Fallout, MassEffect, los juegos de rol desarrollan el cálculo mental, el vocabulario, estimulan la creatividad, además de valores que les permiten socializarse tales como: la empatía, la tolerancia, la responsabilidad, todo esto unido al trabajo en equipo. Cabe destacar que los juegos de rol tienen mucha campo para ser explorado por los docentes, tal es el caso del juego Innov8, el cual ayuda a los estudiantes a formar habilidades en los negocios, a formar jefes, otros juegos orientados con estas características son: Yuti, Regnum y mare Nostrum.

Otra de las bondades que cabe rescatar de los juegos de rol en la adaptabilidad a las necesidades pedagógicas que se van planteando según el momento histórico. 
(Ortega y Pérez, 2011). Juegos de plataforma contribuyen al desarrollo psicomotor y la orientación espacial de los estudiantes, aspecto de gran utilidad en el caso de los más pequeños.

\section{Metodología}

La investigación fue aplicada en el colegio Bethlemitas sede Medellín Colombia, bajo un enfoque mixto (Cualitativo y Cuantitativo), teniendo en cuenta que:

"desde hace varios años creemos firmemente que ambos enfoques, utilizados en conjunto, enriquecen la investigación. No se excluyen ni se sustituyen“. (Hernández, Fernandez y Baptista, 2006).

Este aporte permite afirmar que la elección del método para desarrollar el estudio, realmente brindará información confiable para poder completar la investigación, por tal motivo se debe aprovechar las bondades del enfoque cuantitativo y el enfoque cualitativo.

Los instrumentos aplicados para la investigación fueron dos (2): una prueba objetiva que busca verificar los conocimientos así como las habilidades de las estudiantes en la temática de circuitos eléctricos y electrónica digital, y una prueba Likert orientada a verificar el gusto de las estudiantes y la aplicación que encuentran para el aprendizaje de las ciencias básicas empleadas en la tecnología.

El desarrollo del experimento se aplicó de la siguiente manera: las temáticas de circuitos eléctricos y electrónicos se explicaron en el aula de clase, tanto para el grupo experimental como para el grupo de control, la diferencia se encontraba en que el grupo experimental recibió 3 sesiones de 45 minutos con los siguientes videojuegos:

LiquidMeasure, cuyo objetivo es diseñar y tomar las decisiones adecuadas para no botar ninguna gota de agua, colocando las tuberías y los recipientes de forma correcta, a partir de unas medidas que se van presentando en cada nivel, además el videojuego tiene una gran similitud con la teoría de circuitos eléctricos, donde las tuberías pueden ser miradas como conductores eléctricos, el agua como la corriente eléctrica, y los recipientes como fuentes de voltaje.

Figura 1. Liquid Measure

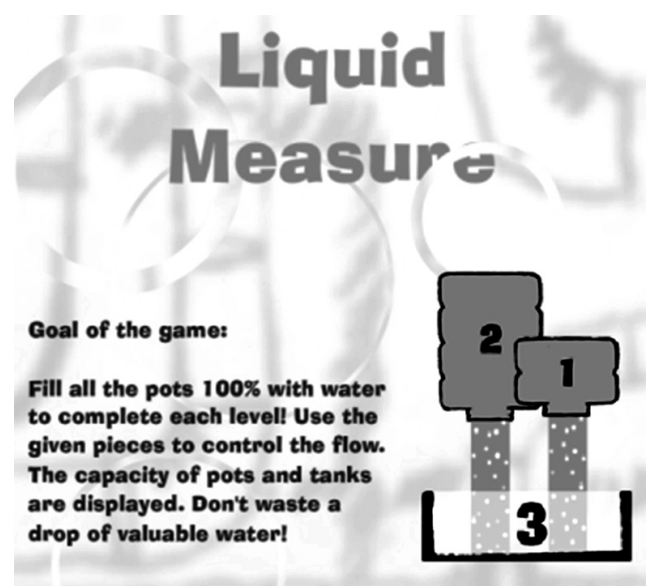

http://www.amigo-play.com/1948/Liquid-Measure-1.html

Cargo Bridge, en este juego se debe construir un puente, de manera que los empleados puedan trasladar las mercancías al otro lado del valle. Para la construcción del puente se debe tener en cuenta que el presupuesto es limitado, por lo que el jugador deberá tomar las mejores decisiones de manera a la hora de construir, además debe tener en cuenta los fundamentos de la física para que la estructura soporte los diferentes pesos.

Figura 2. Cargo Bridge

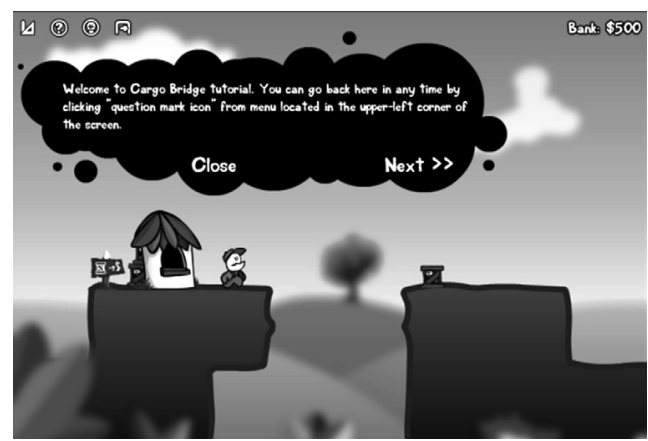

Fuente: http://limexgames.com/studio/games/cargo_bridge 
Tabla 1. Cantidad de estudiantes del grupo experimental y el grupo de control

\begin{tabular}{|c|c|c|c|c|}
\hline Población & $\mathbf{N}^{\circ}$ de estudiantes & Grado & Muestra & $\mathbf{N}^{\circ}$ de alumnos \\
\hline $\begin{array}{c}\text { Estudiantes del grado } \\
\text { décimo colegio Bethlemitas }\end{array}$ & \multirow{2}{*}{102} & $10^{\circ}$ & Grupo de control & 22 \\
\cline { 4 - 5 } & & & Grupo experimental & 22 \\
\hline
\end{tabular}

Fuente: Cálculos de la muesta

Luego de terminar el trabajo con los videojuegos a los dos grupos se les aplicó una evaluación que consta de 16 preguntas de selección múltiple, divididas en 10 preguntas de electricidad, donde se busca verificar los conocimientos básicos en cálculo, diseño y aplicación de los circuitos en serie y en paralelo. Igualmente, se resolvieron 6 preguntas de electrónica digital en las cuales se incluye las compuertas AND, OR y NOT, además de las aplicaciones en el diseño de circuitos combinacionales, herramientas importantes para la construcción de circuitos electrónicos.

Para la confiabilidad de los instrumentos se empleó el alfa de cronbach para la prueba objetiva tanto para el grupo experimental como para el grupo de control, y la prueba de mitades se aplicó al grupo experimental tanto en la prueba objetivo como en la prueba Likert.

\section{Población y muestra}

Población "es un conjunto de medidas o el recuento de todos los elementos que representan una característica común". (Martínez, 2006).

La población que se desea estudiar es de 102 estudiantes del grado decimo pertenecientes al colegio Bethlemitas Sede Colombia- Medellín, con edades comprendidas entre 14 y 17 años de edad, los grupos están divididos en varios niveles $10 \mathrm{~A}, 10 \mathrm{~B}$ y $10 \mathrm{C}$, los cuales cuentan con 35 , 33, y 34 estudiantes respectivamente, esta población fue seleccionada por la accesibilidad que existe para trabajar con ellas en la institución, además de las falencias encontradas en las ciencias básicas y el gusto por el manejo de videojuegos orientados al desarrollo lógico, también se tomó en cuenta el interés por introducir escenarios de aprendizaje más lúdicos y motivadores, los cuales lleven a las estudiantes a prestar más atención e interés por los temas que se desarrollan en clase.

La muestra que se tomo equivale a 44 estudiantes y se dividieron en 2 grupos uno de control y otro experimental, ver tabla 1.

La selección de las estudiantes del grupo de control se realizó de manera aleatoria con las listas del colegio en Excel, el grupo experimental fue seleccionado teniendo en cuenta el bajo rendimiento académico en el área de ciencias básicas.

El muestreo estadístico es un enfoque sistemático para seleccionar unos cuantos elementos (una muestra) de un grupo de datos (una población), a fin de hacer algunas inferencias sobre el tota". (Espagni, Dante, Roldan y López, 2005).

\section{Resultados de la investigación}

Tabla 2. Grupo de Control 22 estudiantes

Resumen estadístico grupo de control

\begin{tabular}{|l|r|}
\hline Resultados \\
\hline Media & 29,1363636 \\
\hline Error típico & 4,23001819 \\
\hline Mediana & 25 \\
\hline Moda & 13 \\
\hline Desviación estándar & 19,840544 \\
\hline Varianza de la muestra & 393,647186 \\
\hline Curtosis & 0,43063744 \\
\hline Coeficiente de asimetría & 1,20950457 \\
\hline Rango & 69 \\
\hline Mínimo & 6 \\
\hline Máximo & 75 \\
\hline Suma & 641 \\
\hline Cuenta & 22 \\
\hline
\end{tabular}

Fuente: Cálculos realizados en Excel 
Tabla 3. Resumen estadístico grupo experimental

\begin{tabular}{|l|r|}
\hline Resultados \\
\hline Media & 40,4545455 \\
\hline Error típico & 5,12431065 \\
\hline Mediana & 31 \\
\hline Moda & 25 \\
\hline Desviación estándar & 24,0351474 \\
\hline Varianza de la muestra & 577,688312 \\
\hline Curtosis & $-1,06818458$ \\
\hline Coeficiente de asimetría & 0,78125774 \\
\hline Rango & 75 \\
\hline Mínimo & 13 \\
\hline Máximo & 88 \\
\hline Suma & 890 \\
\hline Cuenta & 22 \\
\hline
\end{tabular}

Fuente: Cálculos realizados en Excel

Tabla 4. Resumen estadístico prueba Likert

\begin{tabular}{|l|r|}
\hline Resultados \\
\hline Media & 4,68636364 \\
\hline Error típico & 0,05397428 \\
\hline Mediana & 4,7 \\
\hline Moda & 4,8 \\
\hline Desviación estándar & 0,25316182 \\
\hline Varianza de la muestra & 0,06409091 \\
\hline Curtosis & 1,30105511 \\
\hline Coeficiente de asimetría & $-1,05206404$ \\
\hline Rango & 1 \\
\hline Mínimo & 4 \\
\hline Máximo & 5 \\
\hline Suma & 103,1 \\
\hline Cuenta & 22 \\
\hline
\end{tabular}

Fuente: Cálculos realizados en Excel

\section{Análisis prueba objetiva grupo de control Vs. Grupo experimental}

Al analizar la media aritmética del grupo experimental con un valor aproximado de 40 puntos y el grupo de control con un valor de 29, se puede mirar que el grupo experimental obtuvo mejor promedio que el grupo de control, este valor se logra evidenciar al observar que en la prueba, 7 estudiantes lograron aprobarla con puntajes de 63, 75, 88, 69, 75, 69 у 75 . Estos valores muestran que la cantidad de estudiantes que ganaron la prueba fue mayor que la del grupo de control que para el caso solo fueron 3 estudiantes.

a) En general el grupo de control obtuvo en las preguntas 1, 2, 3, 4, 6, 8, 9 mayor puntaje que el grupo experimental, estas preguntas requerían de ciertos cálculos matemáticos, y algunos análisis de gráficos, donde la estudiante debía responder que pasa con el circuito bajo determinadas condiciones.

b) En la pregunta 7 la cantidad de estudiantes del grupo de control, que respondió de forma correcta fue igual al grupo experimental esta pregunta tenía que ver con tener claro el concepto de circuito en paralelo, para reconocer en un gráfico si las resistencias se encontraban bajo esa configuración, adicional a esto esa pregunta fue la que más número de estudiantes respondió de forma correcta.

c) En las preguntas de la 12 a la 16 se requería construir modelos matemáticos y realizar un análisis de lo que sucede con determinado circuito combinacional; el número de estudiantes del grupo experimental que respondieron de forma correcta las preguntas fue mayor en cada uno de los ítems que en el grupo de control.

\section{Análisis aplicación Prueba Likert}

A partir del resumen estadístico en la prueba Likert se puede observar que la media es 4.7 , un resultado muy alto que indica que en general a las estudiantes les gusta el trabajo con videojuegos, y lo encuentran muy útil para mejorar los índices de promoción en Ciencias Básicas, mejorar el ambiente de aula, y ayudarles a resolver problemas con mayor facilidad.

Estas conclusiones están apoyadas por la desviación estándar, la cual fue de 0.25316182 , resultado que muestra una gran homogeneidad en las respuestas debido a la muy poca dispersión de los datos, y la tendencia a respuesta muy satisfactorias con respecto al trabajo con videojuegos en el aula de clase y su aplicabilidad en las ciencias Básicas. 
Después de haber analizado los resultados obtenidos en la prueba Likert, desde el comportamiento, el compromiso, interés, y la motivación que generó para las estudiantes el haber trabajado con los videojuegos, apoyados por resultados numéricos obtenidos en las pruebas objetivas, y las observaciones desarrolladas mientras las estudiantes se disponían a jugar con Liquid Measure y Cargo Bridge , en las cuales se logró determinar cómo el nivel de dificultad entre un nivel y otro proveen a las estudiantes de herramientas para solucionar niveles más avanzados, por lo tanto este trabajo requería de mayor concentración, análisis lógico y dedicación.

Una consideración adicional a partir de las observaciones realizadas, fue la propuesta de trabajo por parte de las estudiantes, donde ellas plantearon que la clase fue muy divertida, lúdica y motivadora, invitando a seguir desarrollando este tipo de prácticas en la asignatura.

Esta respuesta se encuentra relacionada con los resultados obtenidos al aplicar la prueba Likert a las estudiantes del grupo experimental.

\section{Conclusiones}

Uno de los grandes logros obtenidos en la investigación, es observar como las prácticas con los videojuegos Liquid Measurey Cargo Bridge, subieron el nivel de promoción de la prueba objetiva en electricidad y electrónica, aunque el resultado de los promedios, para ninguno de los casos fue satisfactorio, el grupo experimental obtuvo un mejor desempeño, y una mejor resultado a la hora de desenvolverse en la prueba, la cual buscaba verificar como las estudiantes del grupo experimental debían analizar problemas del campo de la electricidad y la electrónica, aplicados a la tecnología, en las cuales lograron construir modelos electrónicos que permitieran dar soluciones a situaciones del mundo real, aplicando la teoría y la práctica.
Un aspecto importante que se encontró al trabajar con videojuegos, fue el desarrollo de cada uno de los niveles partiendo del más sencillo al más complejo, teniendo en cuenta que la solución de los primeros se podía emplear para solucionar niveles más avanzados, en los cuales el estudiante a partir de la práctica, la percepción, el reconocimiento de los espacios, realizaba descubrimientos que le permitirían aplicar un determinado procedimiento de forma lógica con un objetivo definido, el cual lo llevaría a tomar decisiones, emplear estrategias para dar solución a los problemas y dar significado al aprendizaje adquirido.

Motivar es una de las actividades más importantes que deben desarrollar los docentes en los estudiantes, y este tipo de estrategias presentaron un trabajo que buscaba interesar, retar las habilidades, conocimientos, el nivel lógico aplicado a un problema de modelamiento, convirtiendo un ejercicio de diversión en un trabajo de producción intelectual, el cual facilitó mejorar su desempeño en una prueba de conocimiento en electricidad y electrónica. Por tal motivo el diseñar un escenario lúdico de aprendizaje puede promover mejores niveles de comprensión en el área de las ciencias exactas, mejorando los niveles de promoción estudiantil, la motivación y el interés por la asignatura.

Uno de los retos más grandes de los docentes a la hora de trabajar con este tipo de herramienta lúdica es buscar el videojuego que se adapte a las necesidades de la temática que desea trabajar, que sea divertido, que motive a jugar, que rete las habilidades, la lógica, y que de forma gradual presente ejercicios más complejos, los cuales requieran los conocimientos obtenidos del juego anterior para resolver el siguiente. Este trabajo necesita del diseño de objetivos claros e instrumentos de evaluación que permitan verificar si realmente se están alcanzando los niveles de cognición por parte de los estudiantes, además de observar si ellos están disfrutando del placer de aprender. 


\section{Hallazgos}

El grupo experimental que trabajó con videojuegos logró desenvolverse mejor en la prueba de electrónica que el grupo de control, la cual buscaba verificar habilidades de modelamiento, más que habilidades en el desarrollo de cálculos matemáticos; las preguntas estaban diseñadas de forma tal que se debían analizar secuencias en planos electrónicos, buscando determinar la función matemática que permitiera describir el comportamiento del circuito. Otras preguntas estaban orientadas a la resolución de situaciones en las cuales se plantean problemas de control electrónico, donde se debía encender un motor o una alarma a partir de una determinada combinación.

El grupo experimental en el desarrollo de las 10 preguntas que corresponde a la parte de electricidad obtuvo un puntaje de 39,5454545 y el grupo control de un puntaje de 39,0909091, la diferencia no es significativa pero se logra observar como el trabajar con videojuegos realmente puede mejorar los niveles de promoción de la institución, en la asignatura de física.

Durante el desarrollo del trabajo con los videojuegos en el aula de clase, se logró percibir en las estudiantes, placer, gusto, pasión y agrado por el trabajo que se encontraban desarrollando; los niveles con alta complejidad las llevaba a buscar la solución por sus propios medios, luego preguntarle a una compañera o buscar al docente para que les diera una idea de cómo desarrollarlo, en ningún momento solicitaron la solución, en este contexto se dio un espacio de zona de desarrollo próximo entendido como:

"la distancia entre el nivel de desarrollo actual, determinado por la resolución independiente de un problema y el nivel de desarrollo potencial, determinado a través de la resolución de ese problema bajo la guía del adulto o en colaboración con un compañero más capaz" (Vygotsky, 1978/1986).
Este práctica convirtió el escenario de clase en un espacio colaborativo, en el cual buscaban solucionar los diferentes problemas que se les presentaban, partiendo de la habilidad que tenían para resolverlo, y aprovechando los conocimientos o experiencias de otras compañeras, lograban diseñar una idea de cómo podrían solucionar el nivel.

La imposibilidad de resolver un ejercicio genera ansiedad, frustración, desmotivación, disminuyendo el interés y llevando las estudiantes a desarrollar una actividad diferente a la planteada, como realizar un trabajo de otro docente, estudiar para una prueba, o ingresar a Facebook, revisar correo u otro tipo de actividades que en general son prohibidas en clase.

Ortega y Sánchez (2004), la desmotivación se explica como la desgana y la falta de implicación e interés por parte del alumnado en los procesos de enseñanza - aprendizaje que dificultan la labor del maestro y afectan el clima de convivencia en el aula, apareciendo conflictos, lucha de intereses o bajo rendimiento académico.

Con el trabajo desarrollado se encontró que las estudiantes se dedicaron a los videojuegos, y ninguna intentó realizar una actividad diferente a la planteada, durante el proceso se observó concentración, una gran motivación e interés, al terminar la hora de clase, algunas estudiantes plantearon que este tipo de actividades eran muy agradables y productivas en sus procesos de aprendizaje, asimismo el ambiente de aula y la convivencia durante las prácticas fueron excelentes, se notó el respeto, el silencio, la atención y el interés para desarrollar las actividades.

"Los videojuegos proporcionan nuevas formas de aprendizaje e información, además de entretener y distraer. Potencian, también, la concentración y la atención, además de ayudar a construir las habilidades físicas y psicomotoras que el niño y del adolescente necesitan en esa etapa de su vida."(Marín y García, 2005: 117). 
La autonomía desarrollada por las estudiantes durante el trabajo fue excelente, la labor del docente fue de espectador y de guía, en ningún momento impartió clase explicando cómo se resolvía alguno de los niveles, solo dio unas indicaciones generales de los lugares donde se puede encontrar los videojuegos. Las estudiantes ingresaron a las páginas, leyeron las instrucciones y analizaron lo que debían hacer para pasar de un nivel a otro, teniendo en cuenta que la información de las explicaciones se encontraba en inglés, lo cual no fue un obstáculo para ellas, porque la parte gráfica y las experiencias con otros videojuegos les ayudó a comprender la finalidad de este.

Durante el trabajo con videojuegos las estudiantes fueron respetuosas entre ellas, dentro de un escenario de sana convivencia, en el cual no se tenía que corregir comportamientos, posturas, ni malas palabras, porque la actividad lúdica que estaban realizando realmente les proporcionaba diversión y esparcimiento, además del entretenimiento que convertía las clases de 55 minutos, en espacios de esparcimiento lúdico.

\section{Recomendaciones}

Una de las recomendaciones acerca del trabajo con videojuegos se puede observar desde la aplicabilidad y el interés que promueve en los estudiantes, por tal razón es importante construir una propuesta curricular, con una serie de prácticas que inviten al docente a innovar en su trabajo educativo, y quitar el mito que se tiene acerca del ocio que generan este tipo de juegos, el secreto está en la buena elección del videojuego y en la serie de actividades que permitirán sustentar las prácticas que se desarrollaran en clase.

Este tipo de herramientas promueve mejores prácticas de convivencia, las cuales parten del respeto por las ideas, propuestas, y las soluciones que plantean las compañeras, esta situación mejora el ambiente de aula que es una de las metas que ha buscado la institución durante mucho tiempo, todo esto se logra al mantener concentradas e interesadas las estudiantes por una actividad y lo más importante al mejorar la interacción entre ellas.

Para el desarrollo de este tipo de prácticas es importante contar con una sala de computadoras adicional, la razón los docentes necesitan un espacio donde puedan sacar a las estudiantes de los ambientes de aula tradicionales, a un escenario apoyado por una herramienta tecnológicas como la computadora, teniendo en cuenta de no abusar del recurso porque de lo contrario pierde el sentido innovador, y se convierte en una actividad académica la cual deja de motivar a las estudiantes.

Es importante destacar que para el desarrollo de la investigación falto tiempo a la hora de realizar más pruebas, y probar otros videojuegos diferentes a Liquid Measurey Cargo Bridge, analizando el posible impacto en otros grupos diferentes al grado decimo, de forma tal que se pueda determinar el nivel de generalización de la investigación en otros grados de la institución.

La investigación fue desarrollada en el área de ciencias básicas en un tema específico como lo es la electricidad y la electrónica, sería importante explorar si en otras temáticas dentro de la física el impacto es positivo o negativo, teniendo en cuenta que los estudios físicos requieren de habilidades para modelar soluciones, y plantear funciones matemáticas que permitan dar solución al fenómeno observado.

Es importante que el docente aproveche los videojuegos más conocidos por todos sus estudiantes, para que después de jugar con ellos, analizar los valores que se fomenten y debatir en clase sobre ellos. El profesorado debe promover reflexiones y discusiones que permitan que los alumnos comprendan los peligros y las consecuencias que tienen aceptar determinados contravalores que aparecen en los videojuegos. 
Como cualquier juego se puede emplear para generar espacios de socialización de los niños y jóvenes, hay que evitar que la motivación y la diversión que proporcionan los videojuegos, lleven a los estudiantes a un abandono de las actividades que se quieren desarrollar con ellos.

Por tal motivo el docente debe conocer el catálogo de videojuegos disponibles para de esta manera promover situaciones de enseñanza - aprendizaje, a continuación se presentarán algunos criterios para la selección: "La edad real a la que van destinados los juegos ya que las indicaciones de los fabricantes son tan genéricas e indefinidas que tienen escasa utilidad. El tiempo necesario para avanzar en el videojuego ya que hay algunos que requieren muchas horas. Evitar aquellos que presenten contenidos con violencia, el sexismo, el racismo, la intolerancia, etc. La posibilidad de vincular actividades de exploración, de análisis, de síntesis y de evaluación" (Ortega y Pérez, 2011).

Como última recomendación en términos de (Gros, 2006). "Destaca la idea de que el profesorado aproveche los juegos como un material educativo con que trabajar para aprender un contenido curricular específico a partir de la creación de un entorno de aprendizaje que permite enfrentarse con un sistema complejo, multidimensional, multimedia e interactivo, por lo que la incorporación del juego en el aula, permite trabajar con todo el grupo de alumnos a través de grupos cooperativos y discusiones conjuntas que proporcionen espacios de análisis y reflexión crítica del propio entorno utilizado."

\section{Bibliografía}

Alfageme, Begoña y Sánchez, Pedro (2002). Aprendiendo habilidades con video juegos. España: Revista científica de comunicación y educación.ISSN1134 - 3478.p 114 - 119.

Bernat, Antonia. (2008). La construcción de conocimientos y la adquisición de competencias mediante el uso de videojuegos. España: Videojuegos y aprendizaje (pp. 93-112). Begoña. Gros (Ed.),

Carraher, Terrezinha, Carraher, David y Schliemann, Ana (1991). En la vida diez, en la escuela cero. Mexico: Siglo XXI

De la Cruz, Francisco. (2008). Conflictos y resistencias en las aulas. Sevilla: Revista Innovación y experiencias educativas. ISSN 1988-6047, N 10

Etxeberría, Felix. (2000). Videojuegos y educación. COMUNICAR. México: Revista de Educación en Medios de Comunicación.

Ferrer, Santiago. (2008). Los videojuegos. Recuperado de http://ardilladigital.com/DOCUMENTOS/TECNOLOGIA\%20EDUCATIVA/TICS/T8\%20VIDEOJUEGOS/08\%20 LOS\%20VIDEOJUEGOS.pdf

Gómez, María. (2005). Videojuegos y transmisión de valores. España: Revista Iberoamericana de educación. P 10
Greenfield, Patricia. (1989). Mind and media: the effects of television, videogames, and computers. Massachusetts, Harvard University

Hernández, Roberto, Fernández, Carlos y Baptista, Pilar. (2006). Metodología de la investigación. México: McGraw-Hill

Kelly, Jeffey. (1992). Entrenamiento de las habilidades sociales. Bilbao: Biblioteca de Psicología Desclée de Brouwer. 3ra. Edición pag 175.

Lacasa, Pilar. (2002). Cultura y Desarrollo. En P. Herranz Ibarra, \& P. Sierra García, Madrid: UNED. Cultura y Desarrollo (págs. 17-50).

Lacasa, Pilar., Méndez, Laura., y Martínez, Ruth. (2008). Aprender a contar historias y a reflexionar con videojuegos comerciales. España: Videojuegos y Aprendizaje (pp. 51-72).Begoña. Gros (Ed.),

Lave, Jaen. (1988). Cognition In Practice Mind, Mathematics and Culture in Everyday Life. New York: University Cambridge

Lobato, Clemente. (1998). El trabajo en grupo. Aprendizaje cooperativo en secundaria. Bilbao: Universidad del País de Vasco Servicio Editorial.

Malaga, Ana. (2009). Videojuegos como dispositivos culturales: las competencias 
espaciales en educación. España :Revista Comunicar Número 34 ISSN: 1134-3478; páginas 183-189.

Marín, Verónica y García, María. (2005). Los Videojuegos y su capacidad didáctico formativa. España. Revista de Medios y Educación, Número 26 p 113 - 119

Martinez, Ciro. (2006). Estadística y muestreo. Bogota: Eco Ediciones.

Ortega, Jose y Perez, Alvaro. (2011). El potencial didáctico de los videojuegos:

Palacino, Fredy. (2007). Competencias Comunicativas, aprendizaje y enseñanza de las Ciencias Naturales: un enfoque lúdico. Revista Electrónica de Enseñanza de las Ciencias Vol 6, № 2, p $275-298$.
Romera, Eva, Ortega, Rosario y Monks Claire. (2008). Impacto de la actividad lúdica en el desarrollo de la competencia social. España: Revista internacional de psicología y terapia psicológica p $193-202$

Spagni, Beatriz, Dante, Augusto, Roldan, Gabriela y Lopéz, Mirta. (2005). Estadística Básica Probabilidad. Litoral: Ediciones UNL pag 190.

The Movies, un videojuego que fomenta la creatividad audiovisual. España: Revista Etik@net. Número 11 ISSN: 1695-324X 